FORMATION Formation emploi

Revue française de sciences sociales

109 | janvier-mars 2010

L'orientation scolaire et professionnelle dans un monde incertain

\title{
Comment l'orientation contribue aux inégalités de parcours scolaires en France
}

How guidance contributes to inequalities in school pathways in france

Wie in Frankreich die Ausbildungs- und Berufsberatung zu Ungleichheiten in

der schulischen Laufbahn führt

Cómo la orientación contribuye a las desigualdades de trayectorias escolares en

Francia

Séverine Landrier et Nadia Nakhili

\section{(2) OpenEdition}

Journals

Édition électronique

URL : http://journals.openedition.org/formationemploi/2734

DOI : $10.4000 /$ formationemploi.2734

ISSN : 2107-0946

Éditeur

La Documentation française

Édition imprimée

Date de publication : 15 mars 2010

Pagination : 23-36

ISSN : 0759-6340

Référence électronique

Séverine Landrier et Nadia Nakhili, « Comment l'orientation contribue aux inégalités de parcours scolaires en France », Formation emploi [En ligne], 109 | janvier-mars 2010, mis en ligne le 01 mars 2012, consulté le 30 octobre 2020. URL : http://journals.openedition.org/formationemploi/2734 ; DOI : https://doi.org/10.4000/formationemploi.2734 


\title{
DOSSIER
}

\section{Comment l'orientation contribue aux inégalités de parcours scolaires en France}

\author{
Séverine Landrier et Nadia Nakhili*
}

\begin{abstract}
Chaque palier d'orientation produit des inégalités qui se cumulent. Celles-ci découlent de mécanismes complexes qui concernent aussi bien les aspirations des élèves et leurs choix d'études que leur traitement par le système scolaire.
\end{abstract}

L'ouverture du système d'enseignement secondaire, dans les années 70 , a fait suite au passage au collège unique. Les années 80 , quant à elles, ont été marquées par une massification de l'enseignement secondaire, avec une augmentation des effectifs pour tous les baccalauréats et dans l'enseignement supérieur. Mais si le développement de l'accès à ces niveaux d'enseignement a été mécaniquement égalisateur ${ }^{1}$ (Euriat \& Thélot, 1995 ; Thélot \& Vallet, 2000 ; Selz et Vallet, 2006), à l'intérieur des niveaux d'enseignement persistent des inégalités d'accès selon l'origine sociale, qui se seraient même accentuées avec le mouvement de massification (Merle et Mear, 1992 ; Merle, 1996, 2000 ; Blöss et Erlich, 2000 ; Albouy et Wanecq, 2003). Ces constats sont confirmés par les

\footnotetext{
${ }^{1}$ Avant le mouvement de massification, les catégories sociales défavorisées ayant le moins accès à ces niveaux de formation sont celles pour lesquelles les taux d'accès ont augmenté le plus rapidement. Principalement parce que les catégories sociales favorisées y accédaient d'ores et déjà massivement. Les écarts d'accès entre les différents milieux sociaux se sont donc réduits, ce qui a été égalisateur, même si des écarts demeurent.
}

* Séverine Landrier est chargée d'études à l'Observatoire régional des métiers (Observatoire régional Emploi-Formation en Provence Alpes Côte-d'Azur). Lors de la rédaction de cet article, elle était chargée d'études Céreq au LEST-CNRS (Laboratoire d'économie et de sociologie du travail), université d'Aix-Marseille. Thèmes de recherche : Évaluation des politiques publiques en éducation ; Influence du contexte scolaire dans l'analyse des parcours de formation initiale dans l'enseignement secondaire et supérieur ; Analyse de l'insertion professionnelle des jeunes sortis de l'enseignement supérieur. Elle a notamment publié : avec Chevalier T. et Nakhili N. (2009), " Du secondaire au supérieur : continuités et ruptures dans les conditions de vie des jeunes, un état des savoirs", La Documentation française, Collection "Panorama des savoirs". S. Le BastardLandrier (2005), "L'expérience subjective des élèves de seconde : influence sur les résultats scolaires et les vœux d'orientation ", L'orientation scolaire et professionnelle, juin, vol. 34, n² 2, pp. 143-164.

Nadia Nakhili est maître de conférences en sciences de l'éducation, à l'université Joseph Fourier (Grenoble I) et rattachée au LSE (Laboratoire des 
sciences de l'éducation de Grenoble). Lors de la rédaction de cet article, elle était chargée d'études Céreq au BETA-CNRS (Bureau d'économie théorique et appliquée), université de Strasbourg. Thèmes de recherche Orientation scolaire et aspirations des élèves ; Effets du contexte scolaire sur les inégalités de trajectoires scolaires et professionnelles; Enseignement secondaire et enseignement supérieur. Elle a récemment publié : avec Chevalier T., Landrier S. (2009), "Du secondaire au supérieur : continuités et ruptures dans les conditions de vie des jeunes - Un état des savoirs ", La Documentation française, Collection "Panorama des savoirs". Nakhili N. (2005), "Impact du contexte scolaire dans I'élaboration des choix d'études supérieures. », Revue Éducation et Formation, $n^{\circ} 72$, septembre.

analyses conditionnelles² menées par Duru-Bellat et Kieffer (2008) sur les seuls bacheliers. Finalement, l'objectif de mener 80 \% d'une classe d'âge au niveau baccalauréat et celui, plus récent, d'en conduire $50 \%$ au niveau bac +2 ne se réalisent pas sans distinctions qualitatives au niveau des séries et types de baccalauréat, et en conséquence au niveau de l'accès aux différentes filières de l'enseignement supérieur. Les publics restent typés socialement, comme l’illustrent les chiffres du ministère de l'Éducation nationale. Si les enfants de cadres représentent $15 \%$ des entrants en sixième, ils constituent $55 \%$ des inscrits en première année de classes préparatoires, et inversement, les enfants d'ouvriers représentent $38 \%$ des entrants au collège et ne forment que $9 \%$ des entrants en CPGE - classes préparatoires aux grandes Écoles (Lemaire, 2008).

Ces inégalités de parcours résultent de mécanismes aux effets cumulatifs. Elles trouvent leur origine, d'une part, au sein de différences de réussite scolaire selon les milieux sociaux, que l'on peut qualifier d'inéga-

\footnotetext{
2 Ce type d'analyse de la démocratisation de l'accès à l'enseignement supérieur considère les chances d'accès à l'enseignement supérieur des seuls bacheliers et pas celles de l'ensemble de la population. Ne considérant que la population pouvant théoriquement accéder au niveau supérieur, ces analyses mettent en évidence le réel degré de démocratisation du palier d'orientation considéré. Les analyses de Duru-Bellat et Kieffer (2008) sont menées également au sein de chaque type de baccalauréat, ce qui permet de savoir si, pour l'ensemble des bacheliers généraux par exemple, l'accès à l'enseignement supérieur s'est démocratisé.
}

lités de réussite, et d'autre part, au sein de différences relevant de l'orientation scolaire ${ }^{3}$.

Tout d'abord, l'accès des milieux les plus favorisés aux cursus les plus « élevés », aussi bien d'un point de vue vertical (c'est-à-dire selon le niveau d'études) que d'un point de vue horizontal (c'est-à-dire à niveau donné, dans les filières les plus sélectives et les plus valorisées) peut relever de différences de réussite suivies d’inégalités des possibles. Les enfants de milieu populaire réussissent moins bien que ceux d'origine sociale favorisée, en particulier en raison de difficultés constatées, dès l'entrée au primaire, se cumulant année après année (Duru-Bellat, 2002). Au total, dès la fin du collège, au palier d'orientation de fin de 3 e, les enfants d'origine sociale défavorisée se présentent avec moins d' ' atouts » que leurs homologues d'origine sociale favorisée. Ces différences de réussite entraînent des orientations dans des cursus différents. La voie professionnelle pour les uns, les différentes séries de baccalauréat général et technologique pour les autres. In fine, une fois dans certaines filières plutôt que d'autres, les choix effectués en amont et relevant de la réussite limitent les possibilités associées à la position de l'élève dans les différentes filières hiérarchisées (les possibilités offertes après un baccalauréat technologique diffèrent de celles offertes par un baccalauréat général et $a$ fortiori scientifique). Ceci conduit, à terme, à des inégalités de parcours relevant initialement de seules différences de réussite scolaire.

Mais, à chaque palier d'orientation, des élèves comparables, aux vues de leurs résultats scolaires et des possibilités qui y sont associées, n’accèdent pas aux mêmes filières selon le genre ou l'origine sociale : nombre de travaux ont souligné que la polarisation des enfants de cadres dans la filière scientifique est loin de s'expliquer par leur seul niveau scolaire (Prost, 1986 ; Duru-Bellat \& Jarousse, 1993 ; Duru-Bellat, Jarousse \& Solaux, 1997 ; Jarousse \& Labopin, 1999). Un constat similaire est dressé à l'entrée en CPGE, puisqu'un élève bachelier scientifique n'ayant jamais redoublé a quasiment trois fois plus de chances d'accéder en classe préparatoire s'il est de milieu favorisé plutôt que de milieu modeste (Lemaire, 2004). Dans ce cas, c'est le processus d'orientation qui est

\footnotetext{
${ }^{3}$ Tout au long de cet article, il s'agira strictement d'orientation scolaire pendant la formation initiale et non de l'orientation tout au long de la vie.
} 
à l'origine de ces différences. En effet, l'orientation scolaire peut se définir, pour reprendre Berthelot (1993), comme « le processus par lequel s'opèrent les ajustements nécessaires entre les souhaits exprimés et les possibilités offertes, l'école fournissant institutionnellement à chacun la possibilité de faire le parcours que ses possibilités et ses goûts lui tracent »; dès lors, elle contribue à ces inégalités de parcours scolaire à travers différents mécanismes intervenant, à possibilités équivalentes, d'une part, au niveau des goûts et des souhaits, et relevant, d'autre part, du fonctionnement de l'institution elle-même. Cet article propose de mettre en évidence la contribution de l'ensemble de ces mécanismes aux "inégalités d'orientation » scolaire. Celles-ci sont généralement considérées comme les mécanismes à l'œuvre au cours du processus d'orientation, conduisant à différencier les parcours scolaires des jeunes selon des critères qui ne concernent pas uniquement le niveau de réussite scolaire.

Le premier mécanisme générateur des différences de parcours scolaire relève des différences de choix d'études selon les milieux ou le genre, pour des élèves aux possibilités scolaires comparables. Le système d'orientation français fonde ses décisions en priorité sur la demande des jeunes et de leur famille ; leurs choix sont in fine à l'origine d'une partie des inégalités de parcours scolaire. Cependant, les choix se définissant comme le processus par lequel les individus sélectionnent une option parmi un ensemble de possibilités, d'un point de vue purement conceptuel, il est difficile de considérer que des choix sont inégaux ; ils sont donc théoriquement seulement différents les uns des autres. Mais ces choix étant typés socialement dans un système où les filières choisies ne sont pas sans incidences sur les possibilités qu'elles offrent, le cumul de ces différences aux paliers successifs n'est cependant pas négligeable en termes d'inégalités de parcours.

Le second mécanisme propre à l'orientation, et qui contribue aux inégalités de parcours scolaires, est celui d'une sélection « inégalitaire » ou non méritocratique car empreinte de subjectivité de la part des acteurs de l'orientation qui ne sélectionnent pas uniquement les élèves sur des critères académiques mais également selon des critères sociaux ou de genre. Dans ce cas, l'orientation devient inégalitaire car à préférences équivalentes, le niveau scolaire des élèves n'est pas l'unique critère de sélection. Enfin, différents contextes d'enseignement marquent la demande d'orientation des élèves et la sélection qui a lieu ensuite. Ainsi, selon la classe, l'établissement ou le bassin de formation ${ }^{4}$, les élèves formulent des choix d'études différenciés car situés dans des contextes où les pairs, les enseignants et les palettes d'offre de formation varient. Ensuite, à choix équivalent, ils seront de nouveau plus ou moins sélectionnés selon ces différences contextuelles. Cela peut contribuer à accentuer les différences selon les milieux sociaux. Nous considérons ces choix comme étant à l'origine d'inégalités d'orientation et nous les nommerons inégalités de contexte.

Afin de rendre intelligibles la conjugaison de ces facteurs (les différences de préférences individuelles, les inégalités de sélection et les inégalités relevant de l'établissement scolaire marquant à la fois les préférences et la sélection) ainsi que leur contribution aux inégalités de parcours scolaires, cet article propose une synthèse des travaux qui s'appuient sur des enquêtes quantitatives nationales ou régionales. Cette revue de littérature se limite délibérément aux travaux ayant mobilisé une méthodologie « toutes choses égales par ailleurs ", en particulier à niveau scolaire des élèves identique. En effet, ce type d'analyse permet de démêler les différences d'orientation dues à des préférences individuelles de celles qui s'expliquent par des inégalités de contexte. Si la valeur ajoutée de ce type de travaux consiste à identifier divers mécanismes permettant d'expliquer, en partie tout du moins, les différences et inégalités d'orientation, il s'agit bien là de tendances statistiques moyennes. Autrement dit, elles résument et simplifient la complexité du phénomène expliqué tel qu'il existe, mais le processus d'orientation ne fonctionne pas de façon aussi mécanique. Si les estimations statistiques ont tendance à figer les phénomènes étudiés, notamment les régularités sociales, la diversité des situations est bien réelle ; par exemple, si les jeunes d'origine populaire ont moins tendance que leurs pairs plus favorisés à se

\footnotetext{
4 À l'instar du bassin d'emploi, le bassin de formation constitue une zone géographique de recrutement d'élèves, le plus souvent une académie et ses académies limitrophes, et qui offre un certain nombre de spécialités de formation. Au palier d'orientation posttroisième, et au niveau de l'enseignement supérieur, des disparités apparaissent quant aux filières proposées selon les différents territoires.
} 
diriger vers les filières les plus élitistes, fort heureusement, nombre d'entre eux y parviennent.

La mise en perspective de ces travaux empiriques permet de constater, dans une première partie, que les préférences des élèves et de leur famille marquent la demande d'éducation à tous les niveaux du système d'enseignement. Une analyse du système d'orientation, aux différents paliers, dans un contexte de disparités entre les établissements, met en lumière, dans une seconde partie, leur rôle spécifique sur la demande des familles ainsi que sur l'orientation.

\section{DES CHOIX D'ÉTUDES TYPÉS À TOUS LES NIVEAUX DU SYSTĖME D'ENSEIGNEMENT}

Les jeunes, et leurs familles, doivent effectuer un certain nombre de choix tout au long de leur scolarité. Ils élaborent ces choix en fonction de leurs objectifs, de leurs ressources et de leur position dans le système éducatif. La formalisation d'un projet d'études les conduit alors, à partir de leur vécu et expérience antérieure, à anticiper et à se projeter dans l'avenir en explicitant leurs aspirations (Béret, 1986).

Au moment où les niveaux d'études se démocratisent, la diversification du système d'enseignement secondaire et supérieur, en France, menant à des positions différentes en aval de celui-ci, confère à des choix $a$ priori «horizontaux» des enjeux «verticaux ». À niveau d'études donné, un baccalauréat scientifique ne permet pas de suivre les mêmes études qu'un baccalauréat littéraire, par exemple. Finalement, la démocratisation de l'accès au lycée s'est réalisée au prix du maintien des hiérarchies implicites à travers les séries de baccalauréat et les filières d'enseignement supérieur. En effet, la réforme des sections de baccalauréats généraux et technologiques des années 1992-1995, dont l'objectif était de mettre fin à la hiérarchie des filières et à la suprématie des mathématiques comme instrument de sélection, n’a pas résisté à l'attachement des acteurs, des équipes éducatives comme des élèves, envers cette hiérarchie qui structure le système d'enseignement secondaire et oriente par l'échec les élèves dans les filières les moins bien « cotées ». Il en est de même pour le caractère élitiste des classes préparatoires et des possibilités de parcours d'études supérieures qui en découlent. Si l'ensemble des travaux présentés s'intéresse particulièrement à l'accès inégal des élèves aux différentes filières, en présentant le parcours scientifique au secondaire ou l'accès en classe préparatoire dans l'enseignement supérieur comme une référence, c'est pour mettre en évidence les inégalités sociales d'accès à ces parcours d'excellence; cela ne doit en aucun cas conduire à considérer ces parcours comme une norme ou un objectif commun à chaque élève. Dans ce contexte de hiérarchies implicites, les demandes des familles sont soit stratégiques et distinctives pour les familles les plus informées, soit plus ou moins ambitieuses selon la place que les familles occupent dans la hiérarchie sociale. Un ensemble de travaux français permet de dresser un état des lieux, à chaque palier d'orientation (en fin de 3 e où le système éducatif se scinde en deux voies, la voie professionnelle et la voie générale et technologique ; en seconde, où les élèves accèdent aux différentes séries de baccalauréat ; puis en terminale où les élèves accèdent aux différentes voies de l'enseignement supérieur), de l'ampleur des différences de choix d'études selon des caractéristiques indépendantes du niveau scolaire Ces travaux seront présentés en suivant l'ordre logique des paliers d'orientation dans le système éducatif, d'abord au secondaire, puis à l'accès dans l'enseignement supérieur.

\section{Les choix d'études dans le secondaire}

L'enjeu des choix d'études se fait jour très tôt au sein du collège unique, à travers les différences observées, selon les milieux sociaux, de « choix » de langues et d'options au sein du collège unique. Ces choix s'inscrivent dans une logique pédagogique quand ils reflètent les affinités des élèves avec certaines disciplines ; ils revêtent une dimension formative, mais peuvent également s'inscrire dans une logique sociale et être assimilés à de véritables stratégies quand ils visent à accéder aux meilleures classes ou aux meilleurs établissements (Duru-Bellat \& al., 1997).

Les travaux de la DEPP5 ont montré notamment les différences de choix de l'option allemand ou latin et

\footnotetext{
5 Direction pour l'Évaluation, la Prospective et la Performance du ministère de l’Éducation nationale.
} 
leurs conséquences en termes de contexte de scolarisation (Caille, 1996 ; Cibois, 1996). Ce sont souvent les élèves les plus favorisés socialement ainsi que les meilleurs élèves qui choisissent ces options, à résultats scolaires donnés. Dès lors, les latinistes et les germanistes se retrouvent dans les meilleures classes, ce qui ne sera pas sans incidence ni sur leur réussite, ni sur leur orientation ultérieure (Duru-Bellat et Mingat, 1997).

En outre, l'analyse des paliers d'orientation dans le secondaire permet de dresser un premier constat : les différences d'orientation relèvent, en premier lieu et pour partie, de l'auto-sélection, ou des choix relativement moins ambitieux des jeunes d'origine sociale défavorisée. Un élève s'auto-sélectionne quand il émet des vœux d'orientation moins ambitieux que ce à quoi son niveau scolaire lui permet d'accéder. Ces élèves se situent en marge d'une norme implicite au fonctionnement du système d'orientation, conduisant les jeunes à formuler des vœux les plus élitistes possibles compte tenu de leur niveau scolaire. Or, quand les choix d'études semblent peu ambitieux par rapport aux possibilités scolaires, les conseils de classe entérinent ces choix plus souvent qu'ils ne proposent aux élèves une orientation plus en adéquation avec leurs possibilités. L'interprétation sociologique de l'auto-sélection est variable selon que l'on se positionne en faveur de la théorie de l'acteur rationnel de Boudon (1973) - qui considère que les jeunes d'origine populaire font leur choix à partir d'une estimation coût-avantages prenant en compte notamment les risques pris en cas d'échec et où la rentabilité est perçue différemment selon les milieux sociaux en raison de la position d'origine dans la hiérarchie sociale - ou du côté de la théorie de la reproduction de Bourdieu (1966), dans laquelle les jeunes d'origine populaire auraient intégré, via le jeu de l'habitus, que les positions sociales élevées accessibles via ces filières d'enseignement ne leur sont pas destinées. Ce constat a d'abord été dressé à partir des travaux pionniers de l'IREDU (Institut de recherche sur l'éducation) (Duru-Bellat et Mingat, 1988) au niveau du palier de fin de $5^{e}$ auquel se substitue le palier de fin de troisième ${ }^{6}$. Les chercheurs montraient, à partir

\footnotetext{
6 En 1991, le palier de fin de 5e a été déplacé en fin de 3e ; mais il s'agit toujours, à ce moment-là, du palier d'orientation proposant la plus forte distinction au sein du système éducatif, puisque les élèves s’orientent et sont orientés ensuite vers les voies générales et techniques ou professionnelles.
}

d'une enquête longitudinale auprès de 2500 élèves, que les différences de choix d'orientation pèsent autant que celles de réussite scolaire dans le passage en 4 e générale. D’autres études de la DEPP, portant sur le Panel 1989, et plus récemment sur le panel 1995, soulignent la diversité des demandes d'éducation à la fin du collège : en effet, il ressort pour les élèves du panel 1995, qu’à résultats scolaires équivalents en classe de $3^{e}$ (dont les notes sont comprises entre 9 et 12$)^{7}$, les élèves sont $94 \%$ à solliciter une orientation en seconde générale et technologique quand leur famille est d'origine sociale favorisée, alors qu'ils ne sont que $56 \%$ dans les familles populaires (DEPP, 2003). Les travaux de Grelet (2005) confirment ces tendances en soulignant, à l'inverse, que l'orientation vers des filières professionnelles n'est pas non plus seulement conditionnée par les résultats scolaires.

Après ce palier déterminant, où s'opère la distinction entre enseignement professionnel et enseignement général, un autre choix va différencier les élèves en classe de seconde dans l'enseignement général. Si au collège les choix d'options des familles vont être à l'origine d'une différenciation des contextes d'enseignement, au lycée, les choix d'options - toujours socialement marqués (le latin, entre autres, qui n’a pas de rapport particulier avec la filière scientifique) sont propices à l'accès aux séries de baccalauréat qui seront in fine les plus valorisées à l'entrée dans l'enseignement supérieur. Différentes études dans les académies de Dijon et Versailles pointent des différences sociales dans le choix de la série de baccalauréat (Jarousse et Labopin, 1999 ; Le Bastard-Landrier, 2004). Si la demande d'orientation en première scientifique est systématiquement moins présente pour les enfants d'origine sociale populaire, et cela à résultats scolaires comparables (Le Bastard-Landrier, 2004), ces jeunes sont également ceux qui maintiennent moins que les autres leur vœu provisoire en faveur de

\footnotetext{
7 Dans cette étude, sont comparés des élèves de 3 e dont les notes au contrôle continu du brevet sont comprises entre 9 et 12. Il s'agit d'évaluations en classe, ou de moyennes scolaires, qui certes ne reflètent pas le niveau réel de l'élève puisqu'elles comportent des biais de notation des enseignants, mais qui sont néanmoins pertinentes dans ce cas précis puisqu’il s'agit des notes sur lesquelles se basent, d'une part les élèves et leurs familles pour formuler leurs vœux d'orientation et d'autre part, les commissions d'orientation pour les valider.
} 
la filière scientifique, à l'issue du conseil de classe du second trimestre ${ }^{8}$ (Jarousse et Labopin, 1999).

Le genre constitue également un facteur de différenciation important dans l'orientation au secondaire. L'ensemble des travaux présentés ici soulignent la spécificité des choix des filles (Caille et Lemaire, 2002 ; Paulin, 2008). En troisième, les filles s'orientent toujours majoritairement vers les filières générales et technologiques. En revanche, si elles ne s'auto-sélectionnent pas à l'entrée en seconde générale, elles le font ensuite à l'égard de la filière scientifique (Caille et Lemaire, 2002 ; Le Bastard-Landrier, 2005). Or, si des constats similaires s'observent au moment de l'entrée dans l'enseignement supérieur (Ananian et al. 2005 ; Lemaire 2005 ; Nakhili, 2005), l'entrée en série scientifique aura d'ores et déjà une incidence sur l'orientation post-bac (Baudelot et Establet, 1992 ; Duru-Bellat et al., 1993 ; Merle, 1996).

\section{Différences de position et de choix à l'entrée dans le supérieur}

À l'entrée dans l'enseignement supérieur, les différences de choix d'orientation qui ont précédé prédéterminent largement les choix des futurs étudiants (Lemaire et Leseur, 2005), mêlant ainsi les différences de choix à des différences de possibles. Quand on observe l'adéquation entre les séries de baccalauréat et l'accès à l'enseignement supérieur, il apparaît que le facteur le plus déterminant de l'orientation postbaccalauréat est relatif au type de baccalauréat obtenu (Berthelot, 1989 ; Lemaire, 2004, 2005 ; Nakhili 2005, 2007). Ceci étant, à ce palier, et à possibilités équivalentes, l'origine sociale et le genre viennent à nouveau nuancer cette tendance. Tout se passe comme si les inégalités de choix se poursuivent et se cumulent, même pour les « rescapés de la sélection et de l'auto-sélection ». Un bachelier scientifique à l'heure,

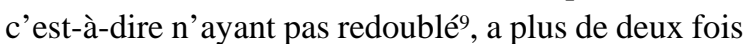

\footnotetext{
${ }^{8}$ Au second trimestre, l'élève et sa famille expriment des souhaits d'orientation à l'aide d'une fiche navette remise par l'établissement : choix de la série de première, redoublement. Le conseil de classe formule ensuite une proposition provisoire d'orientation. Au troisième trimestre, l'élève et sa famille formulent leurs vœux définitifs d'orientation. En juin, le conseil de classe répond par une proposition d'orientation. Si elle est conforme au vœu de l'élève, cette dernière devient une décision d'orientation. Si tel n'est pas le cas, le chef d'établissement prend la décision après un entretien avec l'élève et sa famille.

9 C'est un indicateur courant de réussite scolaire un France.
}

plus de chances de vouloir s’orienter en classe préparatoire quand il est de milieu favorisé que quand il est issu d'un milieu populaire (Nakhili, 2007, 2008).

Si les filles, à caractéristiques scolaires identiques, ont deux fois moins de chances que les garçons d'envisager une filière sélective comme la classe préparatoire (Nakhili, 2005), leurs projets professionnels, également différents de ceux des garçons, semblent expliquer en partie la spécificité de leurs choix (Lemaire, 2005).

Au terme de ce panorama des recherches sur les différences d'orientation relevant des choix socialement et sexuellement marqués des jeunes, à niveau scolaire identique, précisons que ces choix d'orientation s'inscrivent par ailleurs au sein de cadres institutionnels et contextuels contraignants.

\section{UN SYSTÈME ÉDUCATIF QUI CONTRIBUE AUX INÉGALITÉS D'ORIENTATION}

L'influence du contexte scolaire sur les inégalités d'orientation transite d'une part, via les choix et les aspirations des élèves qui s'inscrivent au sein de contextes inégaux, d'autre part, via des mécanismes de sélection pas toujours méritocratiques. Au-delà de ces phénomènes, le fonctionnement du système d'orientation, basé sur la demande des familles, contribue aux inégalités d'orientation en entérinant des vœux typés.

\section{Des « préférences » situées dans des contextes scolaires inégaux...}

À possibilités équivalentes, des élèves de milieux populaires s' « auto-sélectionnent » à tous les paliers d'orientation ; dès lors, ces préférences ne s’inscriventelles pas dans un contexte décisionnel qui découlerait directement de l'organisation du système éducatif et des inégalités qu'elle peut générer ? Aujourd’hui, le constat de différences entre les établissements, en matière de composition sociale ou académique, est avéré ; ces derniers sont typés scolairement, socialement (Trancart, 1998 ; Thomas, 2005), ethniquement (Felouzis, 2003, 2005) et constituent de fait des « micro-milieux » typés. À la ségrégation sociale 
des établissements scolaires s'ajoutent d'autres différences contextuelles. L'offre scolaire de langues et d'options, de séries de baccalauréat ou de formations d'enseignement supérieur, ainsi que la population enseignante, y diffèrent. Ces facteurs interagissent et se cumulent ; par exemple, les lycées qui proposent des classes préparatoires sont ceux qui présentent le recrutement social le plus élitiste (Nakhili, 2005). Les travaux de Dubet (1991) faisaient d'ailleurs état des différences «d'expériences lycéennes » au sein de contextes aussi variables qu'un lycée de centreville avec filières générales et classes préparatoires ou un lycée polyvalent proposant des filières générales, technologiques et professionnelles, situé en périphérie urbaine au sein de laquelle les classes sociales les plus défavorisées sont concentrées.

Au collège comme au lycée, la diversité des conditions d'enseignement, mais aussi des contextes d'information et de décision différents sont plus ou moins propices à la formulation de certains choix d'orientation plutôt que d'autres.

Au collège, les travaux de Duru-Bellat et Mingat (1988) sur le palier de $4^{\mathrm{e}}$ ont mis en évidence des effets contextuels sur les aspirations des familles. Ils constatent qu'à résultats scolaires donnés, la demande moyenne de passage en quatrième est plus importante quand la proportion d'élèves de milieu favorisé est élevée. On regrette cependant que cette recherche n’ait pas quantifié l'impact de la composition sociale sur les vœux des familles, à origine sociale des familles donnée. Les travaux ultérieurs, consacrés à « l'effet établissement », corrigent ce biais, mais seulement au niveau du lycée.

En seconde, les travaux de Le Bastard-Landrier (2002, 2004) mettent en évidence, à partir d'une enquête menée dans 32 lycées des académies de Dijon et Versailles, que la demande d'orientation en $1^{\text {re }} S$ (scientifique) est liée au contexte de scolarisation, à savoir à l'établissement ainsi qu'à la composition sociale de son public d'élèves, et ce à caractéristiques individuelles sociales et scolaires contrôlées. L'influence de la composition sociale du public sur les aspirations scolaires, en seconde, semble confirmée par les résultats d'une étude exploratoire de DuruBellat et al. (2004), conduite dans les mêmes établissements. Les élèves interrogés en classe de seconde se montrent significativement plus ambitieux, à origine sociale comparable, dans un contexte social favorisé ou de bon niveau académique. Concernant l'impact de l'offre de formation des établissements sur les choix des élèves, les travaux de Le Bastard-Landrier (2004) montrent qu'en classe de seconde les élèves scolarisés au sein de lycées proposant également un enseignement de type technologique ont (à niveau scolaire donné) une propension inférieure de $11 \%$ aux autres à envisager une orientation en première scientifique.

Au niveau du palier d'orientation post-secondaire, les résultats de Lemaire (2005) confirment l'existence d'un effet de l'offre de formation sur la demande, puisque l'auteur constate qu'un bachelier généraliste, à caractéristiques identiques, se dirige plus fréquemment en classe préparatoire et un bachelier technologique en STS (sections de techniciens supérieurs), si cette formation est présente dans l'établissement qu'il fréquente en terminale. Dans ce sens, les travaux de Nakhili (2005 ; 2007) montrent également que, quelle que soit la composition sociale de l'établissement, un élève, à résultats scolaires et origine sociale donnés, a près de deux fois plus de chances de s'orienter en classe préparatoire si son établissement en comporte une. Par ailleurs, les travaux portant sur le Panel 1995, ainsi que des données IPES ${ }^{10}$ de la DEPP mettent en évidence des effets spécifiques de la composition sociale des lycées sur les aspirations scolaires des élèves de terminale en termes de nombre d'années d'études envisagées, et de type de formation visé : à origine sociale donnée et à profil scolaire comparable, les lycéens ont toujours des projets d'études plus longs et envisagent d'autant plus une orientation en classe préparatoire qu'ils sont dans un établissement à recrutement social favorisé.

Si l'ensemble des facteurs liés au contexte semble autant affecter les choix d'orientation, les travaux de Le Bastard-Landrier (2002), en classe de seconde, et ceux de Nakhili (2007), en classe de terminale, mettent également en évidence les effets cumulés de l'origine sociale et du contexte de scolarisation : si en classe de seconde, l'impact de l'origine sociale est plus marqué que celui du contexte, en classe de terminale, l'impact du contexte de scolarisation est toujours au moins d'importance égale à celui de l'origine sociale, à laquelle il se cumule ou qu'il compense. Par

\footnotetext{
${ }^{10}$ Indicateurs pour le pilotage des établissements du second degré (ministère de l’Éducation nationale).
} 
exemple, le niveau moyen d'études supérieures visé par un jeune d'origine sociale favorisée, scolarisé dans un établissement au public d'élèves majoritairement défavorisé, est inférieur à celui d'un enfant d'origine sociale défavorisée, scolarisé au sein d’un établissement au public d'élèves majoritairement favorisé. Cette tendance est observée quels que soient l'âge, le sexe et le niveau scolaire des élèves (Nakhili, 2007, 2008). Au-delà de ces tendances statistiques, précisons qu'un élève d'origine sociale favorisée a logiquement plus de chances d'être inscrit dans un établissement scolaire au public majoritairement favorisé. Ainsi, dans une réalité pas toujours égale par ailleurs, les incidences de l'organisation des établissements du secondaire cumulant ségrégation sociale et inégalités d'offre de formation ne sont pas à négliger en termes de cumul des inégalités sociales.

Ces conclusions invitent évidemment à rechercher l'origine de ces différences d'aspirations liées à l'environnement scolaire. La littérature suggère un certain nombre d'hypothèses explicatives qui sont relatives, d'une part, aux interactions au sein des groupes de pairs, d'autre part, aux pratiques et représentations enseignantes. En effet, certains travaux sociologiques (Van Zanten, 2001 ; Duru-Bellat et al., 2004) traitant notamment de la diversité des pratiques enseignantes selon leur contexte d'exercice, invitent à penser qu'elles influencent sensiblement les aspirations des élèves. Duru-Bellat et al. (2004) montrent par exemple que les enseignants des établissements qui scolarisent un public socialement plutôt défavorisé sont moins optimistes quant aux possibilités de réussite au baccalauréat ou de poursuite d'études supérieures de leurs élèves que les autres. Même si ces constats masquent des différences de réussite scolaire selon les lycées, on peut supposer que ces enseignants ont moins tendance à inviter leurs élèves à s'engager dans des études longues, sélectives ou élitistes. Le rôle de l'enseignant dans le choix de l'orientation scolaire des élèves pose, en filigrane, la question de la qualité de l'information diffusée auprès des élèves et de leur famille selon les établissements (en partie via les conseillers d'orientation). Ce facteur étant susceptible de contribuer à l'explication de l'influence de l'offre de formation dans les choix des jeunes.

Au final, si on compare des élèves aux caractéristiques sociales et scolaires identiques qui réalisent l'ensemble de leur carrière scolaire au sein de contextes socialement typés, ces effets, mesurés à chaque palier d'orientation et dont l'analyse laisse apparaître les mêmes phénomènes, s'ajoutent et pèsent au total de façon importante.

\section{Eł une sélection pas toujours méritocratique...}

Enfin, au-delà de ces contextes scolaires qui renforcent l'hétérogénéité socialement marquée des choix des élèves, s'ajoutent les effets de la sélection par les commissions d'orientation et les établissements qui, à demande donnée, ne dépend pas uniquement des critères de réussite scolaire des élèves, comme pourrait l'exiger un véritable système méritocratique (Besnard, 1993.

Au collège, Duru-Bellat et Mingat (1988) montrent, dans leur étude sur le palier de 5 e, que les établissements qui scolarisent un public plutôt populaire étaient, pour des élèves comparables au regard de leurs caractéristiques scolaires, plus sélectifs que les collèges au public favorisé. En classe de seconde, si les élèves d'origine favorisée ont une probabilité plus importante que leurs pairs de demander une orientation en première scientifique, une fois que leur vœu d'orientation a été formulé, ils ont, toujours à résultats scolaires identiques, une plus grande probabilité d'être affectés au sein de cette filière. Ainsi, les conseils de classe entérinent les différences sociales relevant des vœux formulés par les élèves et leur famille et contribuent à leur accroissement au moment des décisions d'orientation (Le Bastard-Landrier, 2002). Pour fournir un ordre de grandeur, environ un quart des lycées sur les 32 de l'échantillon ${ }^{11}$ ont tendance soit à accroître les différenciations sociales apparues au moment des vœux d’orientation, soit à en générer de nouvelles (Le Bastard-Landrier, 2004). Par exemple, si les filles ont davantage tendance à s'auto-sélectionner par rapport aux garçons à l'égard de l'orientation en première scientifique, elles se voient en outre pénalisées dans leur accès à cette filière par les conseils de classe. Même si elles demandent une orientation en première $\mathrm{S}$, à niveau scolaire et origine sociale identiques, les filles ont en moyenne $10 \%$ de chances en

\footnotetext{
${ }^{11}$ Cette recherche a été réalisée à partir d'un échantillon de 32 lycées, dont 27 dans l'académie de Dijon et 5 dans l'académie de Versailles.
} 
moins que les garçons de voir leur vœu entériné (Le Bastard-Landrier, 2005). Si les pistes du rapport aux disciplines, de la confiance en soi, et de l'anticipation de la vie future entrainant des projets professionnels particuliers peuvent être avancées pour expliquer les différences de choix d'orientation des filles, comment expliquer les raisons justifiant les mesures discriminantes des conseils de classe prises à leur égard ? Une piste possible est la gestion des places dans les autres filières. En effet, cette recherche présente un lycée qui fait office d'exception, et qui oriente davantage les filles en première scientifique car il propose un grand nombre de filières technologiques dans lesquelles les garçons sont massivement affectés. Enfin, toujours dans ce travail, au niveau du palier de seconde, on observe un taux de vœux d'orientation insatisfaits variant de $9 \%$ à $39 \%$, selon les lycées, ainsi qu’une grande variété des pratiques en matière d'orientation des élèves. Si certains établissements entérinent quasi automatiquement les demandes des élèves et de leur famille, d'autres préfèrent proposer à un grand nombre d'entre eux soit de redoubler, soit d'être orientés dans une autre filière que celle qu'ils envisageaient et très exceptionnellement dans une série mieux valorisée. Par exemple, les élèves de seconde orientés dans une filière moins prestigieuse que celle demandée représentent jusqu'à $73 \%$ des élèves dont les vœux n’ont pas été entérinés dans un lycée au recrutement populaire, alors qu'aucun n'est dans ce cas dans un établissement favorisé de centre ville (Le BastardLandrier, 2004). L'orientation apparaît donc comme un processus interne relevant de la politique propre à chaque établissement et qui consiste en partie à gérer la composition du public en répartissant les élèves de façon plus ou moins méritocratique au sein des différentes séries. En effet, l'activité des acteurs de l'orientation s'inscrit dans cette logique de contraintes de flux, et ce à tous les paliers d'orientation (Berthet et al., 2007). C'est ce qui la rend inégalitaire dans la mesure où la gestion des flux ne se fait pas seulement en fonction des critères de réussite scolaire.

Comme le souligne Guichard (1999), l'orientation après le baccalauréat constitue un cas singulier par rapport aux pratiques d'orientation en vigueur dans l'enseignement secondaire. "C'est le seul palier où les procédures d'orientation ne dépendent plus de "propositions" des enseignants des établissements d'origine mais de décisions de jurys de sélection d'établissements d'accueil, ou... du "libre choix" de chacun ». C'est donc davantage la demande qui détermine l'orientation finale, puisque le système d'enseignement supérieur est relativement ouvert ou peu sélectif au moment de l'accès. L'université accueille en proportion la majorité des nouveaux étudiants, et les filières de l'enseignement technique court, à accès réglementé par une sélection théoriquement académique, sont peu sélectives (CNE, 2004). Seules les classes préparatoires affichent une sélectivité marquée. À notre connaissance, aucune recherche sur les inégalités dues à la sélection, à demande donnée, à l'entrée de cette filière, n’a été réalisée. La mise en comparaison de deux études, l'une analysant la demande (Nakhili, 2007, 2008), l'autre l'accès en classe préparatoire (Lemaire, 2004,) sur les mêmes données ${ }^{12}$, apporte un certain éclairage. Si un futur bachelier $\mathrm{S}$ à l'heure a une propension 2,3 fois plus importante d'envisager une classe préparatoire quand il est de milieu social favorisé plutôt que de milieu défavorisé, il aura par ailleurs 2,8 fois plus de chances d'y accéder qu'un homologue de milieu populaire. En d'autres termes, les différences d'accès selon le milieu social sont plus importantes que les différences de demande observées en amont. Ceci étant, une fois un dossier accepté, les nouveaux étudiants sont susceptibles de changer d'avis et de rejoindre une autre filière. À ce moment, il est fort probable que de nouveaux phénomènes d'auto-sélection apparaissent. Une certaine prudence est donc nécessaire dans l'interprétation de ces constats tant qu'une réelle analyse de la sélection en classe préparatoire n’aura pas été réalisée.

\section{Dans un système d'orientation basé sur la demande et qui entérine des choix bien typés...}

Au total, les élèves qui poursuivent leurs études jusqu’à l'enseignement supérieur sont amenés à franchir des paliers d'orientation successifs au cours desquels les différences d'aspirations et les inégalités de sélection décrites tout au long de cet article se sont cumulées.

Les inégalités observées tout au long des parcours scolaires relèvent d'abord de différences de choix

12 Données du Panel 1995 de la DEPP, bacheliers 2002 dans l'enseignement supérieur en 2003. 
émanant des élèves et de leur famille. Ces différences reflètent, d'une part, des degrés d'information et de connaissance du fonctionnement du système éducatif variables selon les milieux sociaux; d'autre part, des préférences, niveaux d'aspirations différents et une capacité à développer des stratégies efficaces en termes de choix d'établissement, de langues vivantes ou d'options, variables. S'ajoutent aux choix différenciés des élèves, des inégalités relevant du fonctionnement même du système éducatif par les disparités qu'il génère. Les choix des élèves s'inscrivent en effet dans des contextes inégaux, d'abord sur le plan de la composition sociale et scolaire du public d'élèves, susceptibles d'influencer leur niveau d'aspiration ; ensuite, sur le plan de l'offre de formation susceptible de créer des disparités d'informations ; et enfin sur le plan des places disponibles au sein de chacune des filières de formation. Finalement, l'organisation du système éducatif crée des contextes différents où les pairs, l'information, les possibles et les équipes pédagogiques varient fortement et exercent un rôle sur l'accès aux différentes filières à chaque palier.

Au-delà de tous ces phénomènes, le fonctionnement même du système d'orientation participe à ces différences et inégalités d'orientation. Les procédures d'orientation sont conçues comme une réponse aux choix des familles et vont, de fait, à leur tour contribuer à l'accroissement des inégalités sociales observées. Tout d'abord, les conseils d'orientation au collège et au lycée entérinent quasi automatiquement les demandes des élèves et, par la-même, ils valident des choix non seulement individuels mais aussi qui résultent de différences entre établissements. En effet, les demandes perçues comme étant irréalistes sont revues à la baisse ; or, dans le cas contraire (c'està-dire quand les élèves émettent des vœux pour une filière dont le niveau scolaire requis est inférieur à leurs résultats), les conseils de classe omettent de proposer une orientation en adéquation avec le niveau scolaire (Hénoque \& Legrand, 2004) des jeunes d'origine populaire qui ont tendance à s'auto-sélectionner (Roux \& Davaillon, 2001), ou une orientation dans une série ou une filière hiérarchiquement mieux située (Le Bastard-Landrier, 2002). En outre, le système d'orientation ne fait pas qu'entériner des préférences hétérogènes, socialement marquées et renforcées au sein de contextes scolaires inégaux; il a également tendance à accroître des inégalités existantes (entre les filles et les garçons, les élèves issus de milieux sociaux différents) et à en générer de nouvelles par le biais d'une sélection pas toujours égalitaire.

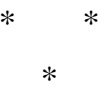

Les élèves et leur famille sont amenés, tout au long de la scolarité secondaire, à effectuer des choix qui conditionnent la suite du parcours de formation. Si au collège et à l'issue de la classe de seconde, l'avis d'orientation du conseil de classe et les procédures d'affectation permettent d'adapter les flux d'élèves à l'offre de formation, ce sont la demande des familles et leur auto-sélection qui prévalent dans le processus d'orientation des jeunes, dès lors qu'elles sont entérinées. Au niveau du palier du baccalauréat, on assiste à une réelle individualisation des parcours scolaires. Si on peut considérer que les inégalités d'orientation relevées à chacun des paliers d'orientation sont d'importance relativement modeste, il importe de souligner qu'au fil du temps, les inégalités observées se cumulent et rendent le processus d'orientation scolaire inapte à assurer non seulement une gestion méritocratique des flux mais aussi une égalité de traitement entre les élèves, principe pourtant réaffirmé, mais toujours démenti, au sein du système éducatif républicain. Les travaux qui se sont attachés à analyser le fonctionnement de l'orientation scolaire, en France, soulignent, de façon récurrente, un certain nombre de limites : si l'orientation se fonde, en partie, sur des critères scolaires, elle n'a de méritocratique que l'apparence puisqu'elle entérine des différences d'orientation marquées par les inégalités sociales entre individus et entre établissements scolaires. Au vu de ces constats, si les choix des individus doivent être respectés au nom du principe de liberté, il s’agirait de les rendre sinon indépendants du milieu social ou du genre du moins indépendants des inégalités créées par les contextes de scolarisation.

Ainsi, les nouveaux dispositifs d'aides à l'orientation, s'ils tentent de respecter les principes d'équité et d'égalité entre les élèves, se heurtent toutefois à des contraintes structurelles inhérentes à la hiérarchie des filières et des baccalauréats. La hiérarchie des filières générales versus professionnelles amène les acteurs 
de l'orientation à diriger par défaut les élèves les plus faibles vers les filières professionnelles. Ce faisant, elle disqualifie la notion de projet et limite les mesures d'éducation à l'orientation prises récemment (module de découverte professionnelle en $3^{e}$; éducation à l'orientation dans les collèges et les lycées, entretien d'orientation obligatoire en 3e, etc.). Quel intérêt les élèves ont-ils à réfléchir à un projet professionnel tant que leur orientation dans les filières techniques et professionnelles se fera par l'échec ? L'impact de ces mesures et du conseil à l'orientation semblent également limités car ils ont plutôt tendance à préparer les élèves aux procédures d'orientation et aux choix à effectuer qu'à les aider à prendre conscience de leurs capacités, à définir leurs centres d'intérêt, à estimer la faisabilité de leur projet, mais également à les former à rechercher de l'information (Legrand, 2005). Quant à l'orientation post-baccalauréat, la généralisation d'un nouveau dispositif d'orientation « active », en
2007, est censée contribuer à la baisse du nombre de sorties sans diplôme de l'enseignement supérieur ; il s’agit d'aider le lycéen à réfléchir, en amont de son inscription dans le supérieur, à ses motivations, à partir d'un avis émis par l'établissement d'enseignement supérieur dans lequel il envisage de s'inscrire. L'élève restant libre de son choix final, l'orientation active ne constitue ni une procédure de sélection, ni une procédure de préinscription, mais se pose en conseil d'orientation. Si ce dispositif doit permettre, sinon un meilleur conseil, du moins un meilleur aiguillage, suite à une première année d'expérimentation, il semblerait que la composition sociale du public estudiantin évolue discrètement en raison d'une autosélection des bacheliers technologiques et professionnels. Il serait intéressant d'en évaluer les effets réels sur les flux étudiants et sur l'avenir de ces bacheliers dans le système d'enseignement supérieur, en matière d'orientation et de réussite.

\section{Bibliographie}

Albouy V. et Wanecq T. (2003), "Les inégalités sociales d'accès aux grandes écoles ", Économie et Statistique, $\mathrm{n}^{\circ}$ 361, pp. 27-52.

Ananian S., Bonnaud A., Lambertyn A. et Vercambre M.-N.(2005), « Les disparités d’orientation au lycée », Éducation et Formations, n 72 pp. 101-113.

Baudelot C et Establet R. (1992), Allez les filles, Paris, Seuil.

Béret P. (1986), « Les projets scolaires : contribution à une théorie de l'acteur dans le système éducatif », Formation Emploi, n 3, pp. 15-23.

Berthelot J.-M. (1989), « Le procès d'orientation de la terminale aux études supérieures ", L'orientation scolaire et professionnelle, vol. $18, \mathrm{n}^{\circ} 1$, pp. 3-22.

Berthelot J.-M. (1993), Ecole, orientation, société, Paris, PUF.
Berthet T., Grelet Y., Romani C. (coord.) (2007), « Le système d'orientation. Entre choix individuels et contraintes d'action publique », NEF-Céreq n 36.

Besnard P. (1993), Dictionnaire de sociologie, Paris, Larousse.

Bloss T. et Erlich V. (2000), « Les nouveaux "acteurs” de la sélection universitaire / les bacheliers technologiques en question ", Revue française de sociologie, vol. $41, \mathrm{n}^{\circ}$ 4, pp. 747-775.

Boudon R. (1973), L'inégalité des chances, Paris, Armand Colin.

Bourdieu P. (1966), "L'école conservatrice. Les inégalités devant l'école et devant la culture ", Revue française de sociologie, vol. 2, n 3, pp. 325-347. 
Caille J.-P. (1996), «Le choix de l'allemand en première langue et la réussite au collège », Éducation et Formations, n ${ }^{\circ}$, pp. 19-38

Caille J.-P. et Lemaire S. (2002), « Filles et garçons face à l'orientation ", Éducation et Formations, $\mathrm{n}^{\circ}$ 63, pp. 111-121.

Cibois P. (1996), "Le choix de l'option latin au collège ", Éducation et Formations, n 48, pp. 39-52.

Chevaillier T., Landrier S., Nakhili N. (2009), Du secondaire au supérieur : continuités et ruptures dans les conditions de vie des jeunes, Un état des savoirs, La Documentation française, Collection « Panorama des savoirs ».

CNE (2004), L'université et l'entrée dans les études supérieures - de Nouveaux espaces pour l'Université, Comité national d'évaluation, Rapport au président de la République.

DEP (2003), « 18 Questions sur le Système Éducatif. Synthèse des travaux de la DEP », Education \& Formations, $\mathrm{n}^{\circ} 66$.

Dubet F. (1991), Les Lycéens, Paris, Seuil.

Duru-Bellat M., Mingat A. (1988), «Le déroulement de la scolarité au collège : le contexte "fait des différences” ", Revue française de sociologie, 29, pp. 649-666.

Duru-Bellat M., Jarousse J.-P. (1993), « La classe de seconde : une étape décisive de la carrière scolaire », Les Cahiers de l'Irédu, n 55, 137 p.

Duru-Bellat M., Jarousse J.-P., Labopin M.-A. et Perrier V. (1993), « Le processus d'auto-sélection des filles à l'entrée en première ", L'orientation scolaire et professionnelle, vol. 22, $\mathrm{n}^{\circ} 3$, pp. 259-272

Duru-Bellat M., Mingat. A. (1997), « La Constitution de classes de niveau dans les collèges; les effets pervers d'une pratique à visée égalisatrice », Revue française de sociologie, vol. 38, octobre-décembre

Duru-Bellat M., Jarousse J.-P., Solaux G. (1997), « S'orienter et élaborer un projet au sein d'un système hiérarchisé, une injonction paradoxale ? L'exemple du choix de la série et de l'enseignement de spécialité en classe terminale ", L'orientation scolaire et professionnelle, Vol. 26, ${ }^{\circ}{ }^{\circ} 4$, pp. 459-482.

Duru-Bellat M., Le Bastard-Landrier S., Piquée C., Suchaut, B. (2004), « Tonalité sociale du contexte et expérience scolaire des élèves au lycée et à l'école primaire ", Revue française de sociologie, 45-3, pp. 441-468.

Duru-Bellat M. (2002), Les inégalités sociales à l'école, Genèse et mythes, Paris, PUF.

Duru-Bellat M., Kieffer A. (2008), « Du baccalauréat à l'enseignement supérieur : déplacement et recomposition des inégalités », Population, vol. 63, n 1 , pp. 123-158.

Euriat M. et Thélot C. (1995), « Le recrutement social de l'élite scolaire en France : évolution des inégalités de 1950 à 1990 », Revue française de sociologie, vol. 36, n 3 , pp. 403-438.

Felouzis G. (2003), "La ségrégation ethnique au collège et ses conséquences ", Revue française de sociologie, 44, 3, pp. 413-448.

Felouzis G. (2005), L'apartheid scolaire. Enquête sur la ségrégation ethnique dans les collèges, Paris, Seuil.

Grelet Y. (2005), «Enseignement professionnel, spécialité de formation et reproduction sociale", Éducation et Formations, nº 72, pp. 125-137.

Guichard J. (1999), « Comment démocratiser la transition lycée-enseignement supérieur », L'orientation scolaire et professionnelle, 28, nº 4, pp. 595-625.

Hénoque, M. \& Legrand A. (2004), L'évaluation de l'orientation à la fin du collège et au lycée : Rêves et réalités de l'orientation, Paris, La Documentation française.

Jarousse J. et Labopin M.-A. (1999), « Le calendrier des inégalités d'accès à la filière scientifique ", L'orientation scolaire et professionnelle, vol. 28, $n^{\circ} 3$, pp. 475-496 
Le Bastard-Landrier S. (2002), Les effets du contexte scolaire sur la réussite des élèves en classe de seconde, Thèse sciences de l'éducation, Dijon, université de Bourgogne, $392 \mathrm{p}$.

Le Bastard-Landrier S. (2004), «Les déterminants contextuels de l'orientation en classe de seconde : l'effet établissement ", Les sciences de l'éducation pour l'ère nouvelle, vol. $37, \mathrm{n}^{\circ} 2$ 2, pp. 59-79.

Le Bastard-Landrier S. (2005), «L'expérience subjective des élèves de seconde: influence sur les résultats scolaires et les vœux d'orientation ", L'orientation scolaire et professionnelle, vol. 34, $\mathrm{n}^{\circ} 2$, pp. 143-164.

Legrand A (2005), «Des arbitrages politiques inachevés : L'orientation scolaire en France », Revue Internationale d'Éducation - Sèvres, $\mathrm{n}^{\circ} 38$.

Lemaire S. (2004), "Que deviennent les bacheliers après leur baccalauréat ? Évolutions 1996-2002 ", Note d'information du MEN, n ${ }^{\circ}$ 04-14

Lemaire S. (2005), «Les premiers bacheliers du panel : aspirations, image de soi et choix d'orientation », Éducation et Formations, $n^{\circ} 72$.

Lemaire S. (2008), « Disparités d'accès et parcours en classes préparatoires », Note d'information $d u$ MEN-08.16.

Lemaire S. et Leseur B. (2005), « Bacheliers S : motivations et choix d'orientation après le baccalauréat », Note d'information du MEN, ${ }^{\circ} 05.15$.

Merle P. et Mear P. (1992), «1986/1990 : démocratisation et/ou hiérarchisation scolaire croissante des publics lycéens ?» Sociétés contemporaines, n० 11/12, pp. 31-52

Merle P. (1996) « Les transformations sociodémographiques des filières de l'enseignement supérieur de 1985 à 1995 : essai d'interprétation », Population, n 6, pp. 1181-1210.

Merle P. (2000), «Le concept de démocratisation de l'institution scolaire : une typologie et sa mise à l'épreuve », Population, n 1, pp. 15-50.
Nakhili N. (2005), «Impact du contexte scolaire dans l'élaboration des choix d'études supérieures des élèves de terminale ", Éducation \& Formations, 72, pp. 165-167.

Nakhili N. (2007), L'environnement scolaire, quels effets sur les aspirations «individuelles»: Le cas de l'entrée dans l'enseignement supérieur, Thèse de doctorat en sciences de l'éducation, Dijon, université de Bourgogne, $302 \mathrm{p}$.

Nakhili N. (2008), «Les aspirations dans leur contexte: une analyse empirique à l'entrée dans l'enseignement supérieur », Actes du colloque «Ce que l'école fait aux individus» - CENS \& CREN - Octobre.

Nakhili N. (2008), « Secondaire-supérieur, quand les choix d'études sont affectés par le contexte de scolarisation », Les cahiers de l'éducation, décembre.

Paulin I. (2008), « Évolution et disparités d'orientation en fin de $3^{\mathrm{e}}$ ", Éducation et Formations, n ${ }^{\circ} 7$, pp. 9-18.

Prost A. (1986), L'enseignement s'est-il démocratisé ?, Paris, PUF.

Roux S. et Davaillon A. (2001), «Le processus d'orientation en fin de troisième: observation des comportements des acteurs et analyse des causalités », Éducation et Formations, n ${ }^{\circ}$ 60, pp. 41-53

Selz M. et Vallet L.-A. (2006), "La démocratisation de l'enseignement et son paradoxe apparent », in Données sociales, La société française, INSEE, pp. 101-107.

Thélot C. et Vallet L.-A. (2000), « La réduction des inégalités sociales devant l'école depuis le début du siècle », Économie et Statistique, n ${ }^{\circ} 34$, pp. 3-32.

Thomas F. (2005), « Disparités entre collèges publics en 2003-2004 », Éducation et Formations, n 71.

Trancart D. (1998), « L'évolution des disparités entre collèges publics ", Revue française de pédagogie, $n^{\circ} 124$, pp. 43-53.

Zanten (van) A. (2001), L'école de la périphérie, Paris, PUF. 
Résumé

\title{
Comment l'orientation contribue aux inégalités de parcours scolaires en France
}

\author{
Séverine Landrier et Nadia Nakhili
}

Dans le contexte actuel de "post-massification » de l'enseignement, des inégalités sociales de parcours scolaires et, in fine, d'insertion professionnelle, demeurent patentes. Si l'insertion des diplômés a ses mécanismes propres, la formation des inégalités sociales de parcours scolaires, en amont, a les siens. L'orientation scolaire en constitue un aspect non négligeable tout au long de l'enseignement secondaire ainsi qu'à l'entrée puis dans l'enseignement supérieur, où les mauvaises orientations sont souvent associées à l'échec ou à l'abandon. Dans ce contexte de réforme de l'orientation, l'article propose de faire le point sur les inégalités scolaires liées à l'orientation et aux différences de choix d'études constatées. II s'agit, à partir d'une revue de la littérature, d'en évaluer l'importance et d'en saisir les mécanismes.

\section{Mots clés}

Orientation scolaire-professionnelle, inégalité sociale, insertion professionnelle, cheminement scolaire, enseignement secondaire, enseignement supérieur, revue de la littérature

Journal of Economic Literature: I 21, J 24 